\title{
Thrombocytosis is a significant indictor of hypercoagulability, prognosis and recurrence in gastric cancer
}

\author{
CHANGYUAN HU $^{1}$, RENPIN CHEN ${ }^{2}$, WENJING CHEN ${ }^{1}$, WENYANG PANG ${ }^{1}$, \\ XIANGYANG XUE ${ }^{3}$, GUANGBAO ZHU ${ }^{1}$ and XIAN SHEN ${ }^{1}$ \\ Departments of ${ }^{1}$ General Surgery and ${ }^{2}$ Gastroenterology and Hepatology, First Affiliated \\ Hospital of Wenzhou Medical University; ${ }^{3}$ Department of Microbiology and Immunology, \\ Wenzhou Medical University, Wenzhou, Zhejiang 325035, P.R. China
}

Received November 14, 2013; Accepted December 10, 2013

DOI: $10.3892 /$ etm.2014.1699

\begin{abstract}
Although thrombocytosis has been reported in a variety of cancer types, the standard of thrombocytosis in gastric cancer (GC) and the association between thrombocytosis and the clinicopathological features of patients with GC remain unclear. In the present study, 1,763 GC patients were retrospectively filtered by preoperative thrombocytosis and compared with control group A $(n=107)$ that had benign gastric lesions and control group B $(n=100)$ that were GC patients with a normal platelet (PLT) count. Associations between clinical variables and preoperative PLT counts were assessed by univariate and multivariate analyses. Kaplan-Meier survival curves and Cox regression were used to evaluate the effect of thrombocytosis on prognosis. Sensitivities and specificities of the PLT counts in predicting recurrence were analyzed via area under the receiver operating characteristic curve (AUROC). The results indicated that the incidence of thrombocytosis in GC patients was higher than in benign gastric lesion patients, with $4.03 \%$ of GC patients having a PLT count $>400 \times 10^{9} / 1$ $(\mathrm{P}=0.014)$ and $12.08 \%$ had a PLT count $>300 \times 10^{9} / 1(\mathrm{P}<0.001)$. For the patients with a PLT count $>400 \times 10^{9} / 1$, the frequency of abnormal PLT counts in GC correlated with tumor size $(\mathrm{P}<0.001)$, tumor, node and metastasis $(\mathrm{TNM})$ classification $(\mathrm{P}=0.002)$, invasive degree $(\mathrm{P}=0.003)$ and $\mathrm{D}$-dimer $(\mathrm{P}=0.013)$ and fibrinogen concentrations $(\mathrm{P}=0.042)$. Tumor size $(\mathrm{P}=0.002)$, TNM classification $(\mathrm{P}<0.001)$ and depth of penetration $(\mathrm{P}=0.001)$ were independent factors for thrombocytosis. However, thrombocytosis functioned as an independent prognostic factor for GC patients with a PLT count $>400 \times 10^{9} / 1$
\end{abstract}

Correspondence to: Professor Guangbao Zhu or Dr Xian Shen, Department of General Surgery, First Affiliated Hospital of Wenzhou Medical University, South Baixiang Street, Shangcai Village, Ouhai District, Wenzhou, Zhejiang 325035, P.R. China E-mail: zgbwmc@126.com

E-mail: shenxian5166@gmail.com

Key words: gastric cancer, thrombocytosis, recurrence, survival analysis (relative risk, 1.538; 95\% confidence interval, 1.041-2.271). In the majority of patients (17/24) with a high preoperative PLT count that decreased to a normal level following resection, PLT levels increased again at recurrence. Sensitivities and specificities of thrombocytosis for recurrence in those patients were 70.8 and $83.3 \%$, respectively (AUROC, 0.847 ; $\mathrm{P}=0.01$ ). Therefore, a PLT count of $400 \times 10^{9} / 1$ is a suitable threshold for defining thrombocytosis in GC. Thrombocytosis was shown to affect the blood hypercoagulable state and also have a negative prognostic value for GC patients. PLT monitoring following surgery was useful to predict the recurrence for specific GC patients that suffered preoperative thrombocytosis but had restored PLT levels following resection.

\section{Introduction}

Thrombocytosis can be induced by a number of primary and secondary factors. Primary thrombocytosis is a type of myeloproliferative disease with $50-70 \%$ of patients exhibiting mutations in the JAK2V617F gene and PLT levels $>600 \times 10^{9} / 1$ and even up to $1,000 \times 10^{9} / 1$ (1). Secondary thrombocytosis is caused by drugs or other diseases, including inflammation, infection, rheumatism, anemia and malignancy, where patients exhibit moderately increased PLT levels that are between 400 and $800 \times 10^{9} / 1$ (2-4). After excluding the diagnosis of essential thrombocythemia, preoperative cancer patients with a PLT count of $>400 \times 10^{9} / 1$ are considered to have secondary thrombocytosis (5-7). However, the threshold remains controversial.

Since the 1980 s, the phenomenon that a comorbidity between blood hypercoaguable state and malignant solid tumors has caused widespread concerned and been increasingly studied. Although the specific mechanism is not completely understood, the association between thrombocythemia and the hypercoagulability state has been confirmed repeatedly (8). Prior to the evidence that malignant tumors lead to coagulation abnormality, Riess firstly proposed the symptoms of unexplained thrombocytosis in malignancies (9). Previously, thrombocytosis, as a paraneoplastic syndrome, has been successively identified in oral squamous cell, renal cell and hepatocellular carcinomas, as well as lung, esophageal, gastric (GC), colorectal, pancreatic and gynecological cancers $(2,10-12)$. 
Table I. Thrombocytosis in patients with gastric carcinomas or benign gastric lesions.

\begin{tabular}{lcrrrrr}
\hline & & \multicolumn{2}{c}{ PLT $>300 \times 10^{9} / 1$} & & \multicolumn{2}{c}{ PLT $>400 \times 10^{9} / 1$} \\
\cline { 3 - 6 } Group & PLT $\leq 300 \times 10^{9} / 1, n$ & $\mathrm{n}$ & P-value & & $\mathrm{n}$ & P-value \\
\hline Gastric carcinoma & 1,550 & 213 & $<0.001$ & & 71 & $0.014^{\mathrm{a}}$ \\
Benign gastric lesions & 106 & 1 & & 0 & \\
\hline
\end{tabular}

${ }^{a}$ Calculated with Fisher's exact test. PLT, platelet. P-value compared the incidence of thrombocythemia with PLT count $\leq 300 \times 10^{9} / 1$ in different groups.

Numerous studies agree that malignancies with unknown causes of thrombocytosis may be a valuable adjuvant parameter in predicting the prognosis of cancer $(2,4,5,11)$. However, the complex effect of thrombocytosis in malignancy has been rarely reported. GC is the second most common malignancy in China, however, there are few specific early clinical manifestations and accurate predictors of prognosis. Therefore, GC patients with a high risk of recurrence may benefit from a reliable predicting indictor. In the present study, associations between thrombocytosis and clinicopathological features, survival time and tumor recurrence were systematically investigated in GC patients. The aim was to provide a new approach in the diagnosis and prognosis of GC.

\section{Methods and methods}

Definition of the blood test indicator. Primary thrombocytosis was eliminated using bone marrow cytomorphological examinations, and thrombocytosis was diagnosed as a PLT count $>400 \times 10^{9} / 1$, which was in accordance with other studies $(5,7,13)$. As the normal PLT count of patients at the First Affiliated Hospital of Wenzhou Medical College (Wenzhou, China) ranges between 100 and $300 \times 10^{\%} / 1$, individuals with a PLT count $>300 \times 10^{9} / 1$ were also analyzed. Carcinoembryonic antigen (CEA), carbohydrate antigen 19-9 (CA19-9), D-dimer, fibrinogen, prothrombin time (PT) and activated partial thromboplastin time (APTT) standards were as follows: $0-5 \mu \mathrm{g} / \mathrm{l} ; 0-37 \mathrm{U} / \mathrm{ml} ; 0-0.5 \mathrm{mg} / 1 ; 2-4 \mathrm{~g} / 1 ; 11-13 \mathrm{sec}$; and 30-45 sec, respectively. Sodium citrate anticoagulated blood samples were collected and analyzed with a COULTER Gen-S automatic blood analyzer (Beckman Coulter, Inc., Miami, FL, USA) and Stago STA-R Evolution automatic coagulometer (Diagnostica Stago, Inc., Beijing, China).

Patients. A total of 1,763 patients who underwent surgical treatment for GC in the First Affiliated Hospital of Wenzhou Medical University between July 2005 and June 2008 were eligible for retrospective review in the study. None of the patients had received radiation therapy or chemotherapy prior to surgery, but had received strict chemical therapy following surgery, according to the National Comprehensive Cancer Network GC guidelines. Histopathological diagnosis of gastric adenocarcinoma was confirmed by the Pathology Department following surgery, according to the World Health Organization's criteria. In total, 71 patients with GC and thrombocytosis were enrolled in study cohort A. These patients had a mean age of 63 \pm 9.15 years (range, 27-84 years).
A total of 213 patients with a PLT count $>300 \times 10^{9} / 1$ were included in study cohort B and these individuals had a mean age of 63 \pm 10.02 years (range, 27-84 years). Control group A comprised 107 patients with benign gastric lesions that had a mean age of $41 \pm 6.33$ years (range, 23-66 years). Control group B comprised 100 cases that had been randomly selected from 1,550 GC individuals with normal PLT counts. The mean age was $66 \pm 8.46$ years (range, $34-88$ years) .

Blood samples were collected from the individuals within 1 week prior to surgery, $\sim 4$ weeks following surgery and during the follow-up period. B-mode ultrasound and physical examinations were performed postoperatively for patients with major complaints to assess the status of deep vein thrombosis (DVT). Imageological examinations, including computed tomography (CT) and enhanced CT scans, were performed to assess recurrence during the follow-up period. Informed written consent was provided by each patient and the study was approved by the Human Research Ethics Committee of the First Affiliated Hospital of Wenzhou Medical University.

Statistical analysis. Associations between thrombocytosis and clinicopathological features were analyzed using the $\chi^{2}$ test, Fisher's exact test, independent sample t-test, Pearson's correlation test and logistic regression analysis. A Kaplan-Meier survival curve and Cox regression model were used to evaluate the clinical significance of thrombocytosis in GC. Area under the receiver operating characteristic curve (AUROC) and the Z-test were applied to analyze the sensitivities and specificities of PLTs in predicting recurrence. $\mathrm{P}<0.05$ was considered to indicate a statistically significant difference. All analyses were performed using SPSS version 16.0 for Windows (SPSS, Inc., Chicago, IL, USA).

\section{Results}

Thrombocytosis in GC patients. As shown in Table I, when compared with control group A (benign gastric lesion, $0 \%$, $0 / 107)$, the morbidity $[4.03 \%, 71 / 1,763 ; 95 \%$ confidence interval (CI), 3.11-4.94] of GC patients with thrombocytosis (PLT $\left.>400 \times 10^{9} / 1\right)$ increased significantly $(\mathrm{P}=0.014)$. This was also observed in patients with a PLT count $>300 \times 10^{\%} / 1(12.08 \%$, 213/1,763; 95\% CI, 10.56-13.60; P<0.001).

Correlation between thrombocytosis and clinicopathological features. As shown in Table II, the incidence of thrombocytosis, defined as a PLT count $>400 \times 10^{9} / 1$, exhibited statistically significant differences when compared with the normal PLT cohort 
Table II. Thrombocytosis and clinicopathological variables in GC.

\begin{tabular}{|c|c|c|c|c|c|}
\hline \multirow[b]{2}{*}{ Variable } & \multirow[b]{2}{*}{ PLT $\leq 300 \times 10^{9} / 1, \mathrm{n}$} & \multicolumn{2}{|c|}{ PLT $>300 \times 10^{9} / 1$} & \multicolumn{2}{|c|}{ PLT $>400 \times 10^{9} / 1$} \\
\hline & & $\mathrm{n}$ & P-value & $\mathrm{n}$ & P-value \\
\hline \multicolumn{6}{|l|}{ Age, years } \\
\hline$<60$ & 39 & 75 & 0.516 & 24 & 0.488 \\
\hline$\geq 60$ & 61 & 138 & & 47 & \\
\hline \multicolumn{6}{|l|}{ Location } \\
\hline Upper third & 20 & 31 & 0.082 & 10 & 0.339 \\
\hline Middle third & 36 & 105 & & 33 & \\
\hline Lower third & 44 & 77 & & 28 & \\
\hline \multicolumn{6}{|l|}{ Tumor size, cm } \\
\hline$<5$ & 58 & 79 & 0.001 & 21 & $<0.001$ \\
\hline$\geq 5$ & 42 & 134 & & 50 & \\
\hline \multicolumn{6}{|l|}{ Type } \\
\hline Adenocarcinoma & 98 & 198 & $0.191^{\mathrm{a}}$ & 67 & $0.263^{\mathrm{a}}$ \\
\hline Squamous carcinoma & 0 & 3 & & 1 & \\
\hline Undifferentiated & 2 & 12 & & 3 & \\
\hline \multicolumn{6}{|l|}{ Degree of differentiation } \\
\hline Well & 15 & 19 & 0.242 & 10 & 0.886 \\
\hline Moderate & 17 & 38 & & 10 & \\
\hline Poor & 66 & 156 & & 48 & \\
\hline \multicolumn{6}{|l|}{ Vascular invasion } \\
\hline Present & 34 & 67 & 0.653 & 29 & 0.422 \\
\hline Absent & 66 & 146 & & 42 & \\
\hline \multicolumn{6}{|l|}{ Perineural invasion } \\
\hline Present & 37 & 80 & 0.924 & 26 & 0.959 \\
\hline Absent & 63 & 133 & & 45 & \\
\hline \multicolumn{6}{|l|}{ Lymphatic invasion } \\
\hline Present & 71 & 174 & 0.172 & 57 & 0.168 \\
\hline Absent & 29 & 49 & & 14 & \\
\hline \multicolumn{6}{|l|}{ TNM classification } \\
\hline $\mathrm{I}$ & 21 & 12 & $<0.001$ & 1 & $0.002^{\mathrm{a}}$ \\
\hline II & 21 & 49 & & 16 & \\
\hline III & 44 & 105 & & 39 & \\
\hline IV & 14 & 47 & & 15 & \\
\hline \multicolumn{6}{|l|}{ Depth of penetration } \\
\hline $\mathrm{T} 1$ & 18 & 10 & $<0.001$ & 1 & $0.003^{\mathrm{a}}$ \\
\hline $\mathrm{T} 2$ & 4 & 15 & & 5 & \\
\hline T3 & 53 & 97 & & 37 & \\
\hline $\mathrm{T} 4$ & 25 & 91 & & 28 & \\
\hline \multicolumn{6}{|l|}{ Distant metastasis } \\
\hline Present & 86 & 166 & 0.093 & 56 & 0.221 \\
\hline Absent & 14 & 47 & & 15 & \\
\hline \multicolumn{6}{|l|}{ CEA } \\
\hline Abnormal & 23 & 43 & 0.570 & 22 & 0.305 \\
\hline Normal & 77 & 170 & & 49 & \\
\hline \multicolumn{6}{|l|}{ CA19-9 } \\
\hline Abnormal & 25 & 55 & 0.877 & 19 & 0.795 \\
\hline Normal & 75 & 158 & & 52 & \\
\hline
\end{tabular}

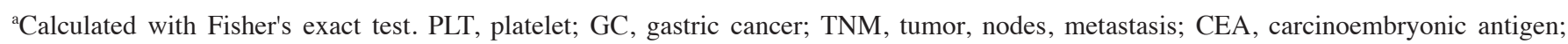
CA19-9, carbohydrate antigen 19-9. P-value compared the incidence of thrombocythemia with PLT count $\leq 300 \times 10^{9} / 1$ in different groups. 
A

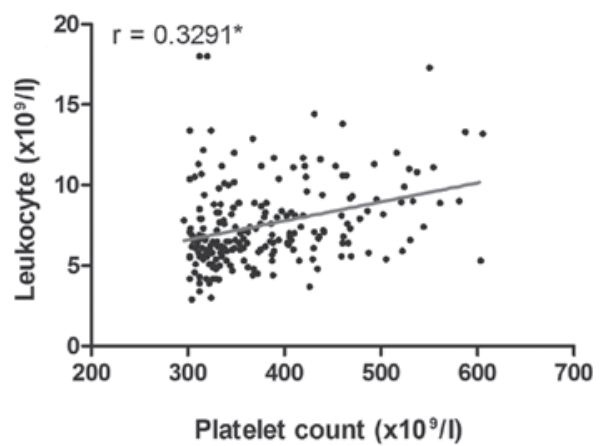

C

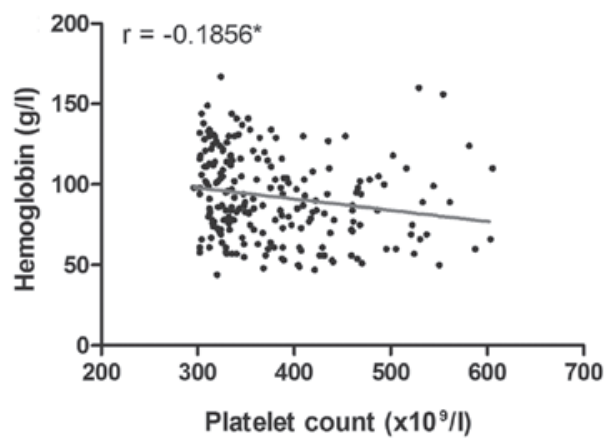

B

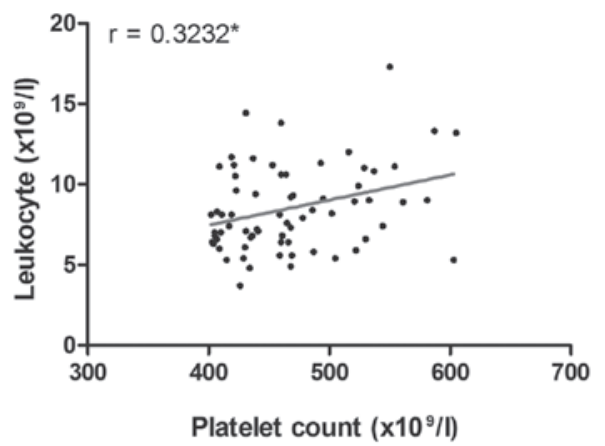

D

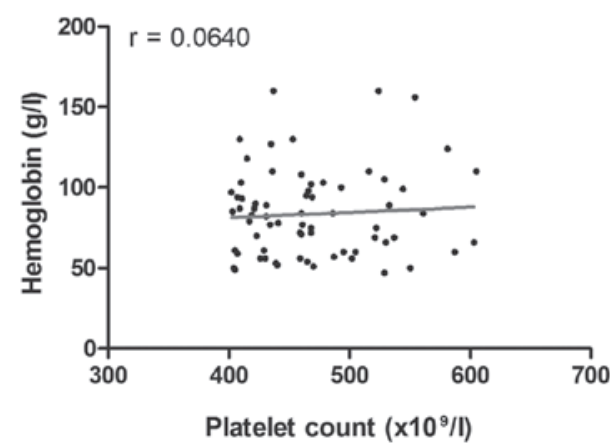

Figure 1. Linear correlation analysis. Correlations between leukocyte counts and $(\mathrm{A})>300 \times 10^{9} / 1(\mathrm{P}<0.001)$ and $(\mathrm{B})>400 \times 10^{9} / 1(\mathrm{P}=0.006) \mathrm{PLT}$ counts Correlation between hemoglobin concentration and $(\mathrm{C})>300 \times 10^{9} / 1(\mathrm{P}=0.006)$ and $(\mathrm{D})>400 \times 10^{9} / 1(\mathrm{P}=0.599) \mathrm{PLT}$ counts. $\mathrm{PLT}$, platelet.

in tumor size $(\mathrm{P}<0.001)$, tumor, node and metastasis $(\mathrm{TNM})$ classification (particularly for phase $\mathrm{I}, \mathrm{P}=0.002$ ) and depth of penetration $(\mathrm{P}=0.003)$. No statistically significant differences were identified in age, tumor location, type, degree of differentiation, vascular invasion, perineural invasion, lymphatic invasion, distant metastasis or tumor markers CEA/CA19-9 $(\mathrm{P}>0.05)$. The same result was verified in patients with a PLT count $>300 \times 10^{9} / 1$ with regard to tumor size $(\mathrm{P}=0.001)$, TNM classification $(\mathrm{P}<0.001)$ and penetration $(\mathrm{P}<0.001)$. Analysis of enumeration data revealed that no statistical significance existed between thrombocythemia and clinicopathological features in the cancer group (data not shown). In addition, to exclude the interaction between tumor size, type, differentiation, lymphatic invasion, penetration and TNM classification, risk assessments were used to measure the effect of these independent variables on thrombocytosis. Tumor size $[\mathrm{P}=0.002$; odds ratio (OR), 2.179; 95\% CI, 1.347-3.526], TNM classification $(\mathrm{P}<0.001$; OR, 1.763; 95\% CI, 1.317-2.360) and depth of penetration $(\mathrm{P}=0.001$; OR, 1.643; 95\% CI, 1.232-2.191) functioned as moderate positive factors for the occurrence of thrombocythemia.

The mean pretreatment PLT count for thrombocytosis patients was $469.23 \pm 53.99 \times 10^{9} / 1$ (range, $402-605 \times 10^{9} / 1$ ), the leukocyte count was $8.54 \pm 2.61 \times 10^{9} / 1$ (range, $\left.3.7-17.3 \times 10^{9} / 1\right)$ and the hemoglobin concentration was $83.51 \pm 26.68 \mathrm{~g} / 1$ (range, 47-160 g/l). The mean PLT count for GC patients that had preoperative PLT levels $>300 \times 10^{9} / 1$ in study group B was $379.92 \pm 73.23 \times 10^{9} / 1$ (range, 302-605 $\times 10^{9} / 1$ ), while the leukocyte count was $7.81 \pm 3.95 \times 10^{9} / 1$ (range, $2.9-20.6 \times 10^{9} / 1$ ) and the hemoglobin concentration was $92.61 \pm 27.14 \mathrm{~g} / 1$ (range, 44-167 g/l). A significant positive linear correlation was identified between the PLT and leukocyte counts when the PLT count was $>300$ or $400 \times 10^{9} / 1(\mathrm{r}=0.3291 ; \mathrm{P}<0.001$; and $\mathrm{r}=0.3232$; $\mathrm{P}=0.006$, respectively; Fig. $1 \mathrm{~A}$ and $\mathrm{B}$ ). However, a correlation between the PLT count and hemoglobin concentration was only verified in patients with a PLT count $>300 \times 10^{9} / 1$ ( $r=-0.1856 ; \mathrm{P}=0.006$; Fig. $1 \mathrm{C}$ ), since $\mathrm{P}=0.599$ for patients with a PLT count $>400 \times 10^{9} / 1$ (Fig. 1D).

Correlation between thrombocytosis and the blood hypercoagulable state. Although ultrasonic examinations did not reveal any statistically significant differences between the control group and study cohorts with PLT count $>300 \times 10^{9} / 1$ or $400 \times 10^{9} / 1(\mathrm{P}=0.444$ and 0.083 , respectively), DVT was more likely to affect tumor patients with thrombocytosis $(7.04 \%$, 5/71) than patients with a PLT count $>300 \times 10^{9} / 1(3.29 \%$, 7/213). Abnormal D-dimer and fibrinogen concentrations occurred more frequently in patients with thrombocytosis (PLT $>400 \times 10^{9} / 1 ; \mathrm{P}=0.004$ and 0.013 , respectively), but no statistically significant differences were observed when the threshold was defined as $300 \times 10^{9} / 1$. In addition, no correlation was observed between the occurrence of anomalous PLT counts and decreased PT/APTT (Table III). As aforementioned, the enumeration data of thrombocytosis demonstrated that no statistical significance was present between the cancer groups with regard to blood hypercoagulability (data not shown).

Survival analysis in GC patients with thrombocytosis. The overall 5-year survival rate of tumor patients with thrombocytosis (PLT $>400 \times 10^{9} / 1$ ) was $16.90 \%$, while the survival rate was $31.00 \%$ in individuals with a normal PLT count. Median survival times were 15 and 24 months, respectively $(\mathrm{P}=0.008$, as determined by the logrank test; Fig. 2A). However, when compared with control group B, individuals 
Table III. Thrombocytosis and coagulation markers in GC.

\begin{tabular}{|c|c|c|c|c|c|}
\hline \multirow[b]{2}{*}{ Variable } & \multirow[b]{2}{*}{$\mathrm{PLT} \leq 300 \times 10^{9} / 1, \mathrm{n}$} & \multicolumn{2}{|c|}{$\mathrm{PLT}>300 \times 10^{9} / 1$} & \multicolumn{2}{|c|}{$\mathrm{PLT}>400 \times 10^{9} / 1$} \\
\hline & & $\mathrm{n}$ & P-value & $\mathrm{n}$ & P-value \\
\hline \multicolumn{6}{|l|}{ PT } \\
\hline Decreased & 8 & 28 & 0.158 & 12 & 0.071 \\
\hline Normal & 90 & 175 & & 57 & \\
\hline \multicolumn{6}{|l|}{ APPT } \\
\hline Decreased & 11 & 30 & 0.465 & 13 & 0.204 \\
\hline Normal & 83 & 172 & & 56 & \\
\hline \multicolumn{6}{|l|}{ Fibrinogen } \\
\hline Normal & 83 & 158 & 0.061 & 51 & 0.042 \\
\hline Increased & 15 & 52 & & 20 & \\
\hline \multicolumn{6}{|l|}{ D-dimer } \\
\hline Normal & 87 & 172 & 0.172 & 51 & 0.013 \\
\hline Increased & 13 & 41 & & 20 & \\
\hline \multicolumn{6}{|l|}{ DVT } \\
\hline Present & 1 & 7 & $0.444^{\mathrm{a}}$ & 5 & $0.083^{\mathrm{a}}$ \\
\hline Absent & 99 & 206 & & 66 & \\
\hline
\end{tabular}

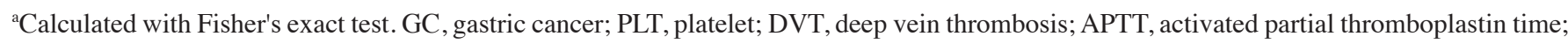
PT, prothrombin time. P-value compared the incidence of thrombocythemia with PLT count $\leq 300 \times 10^{9} / 1$ in different groups.

A

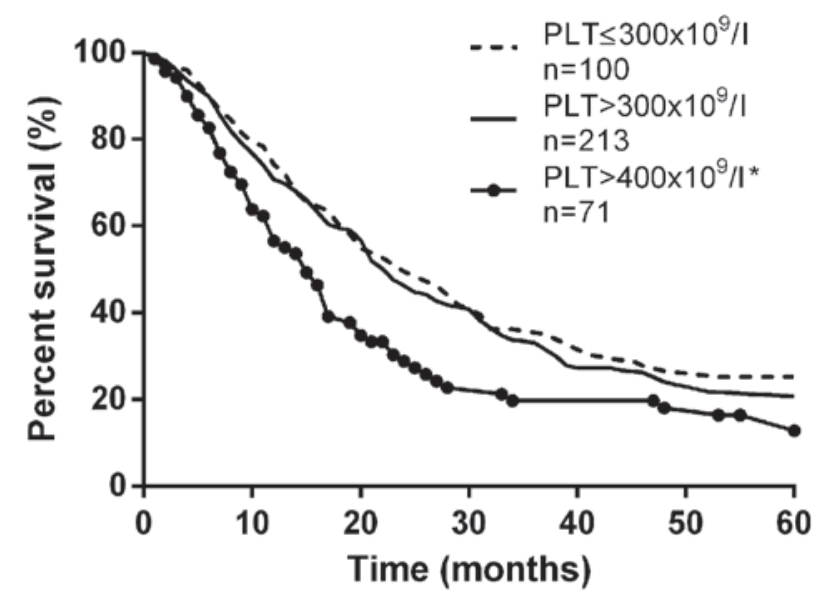

B

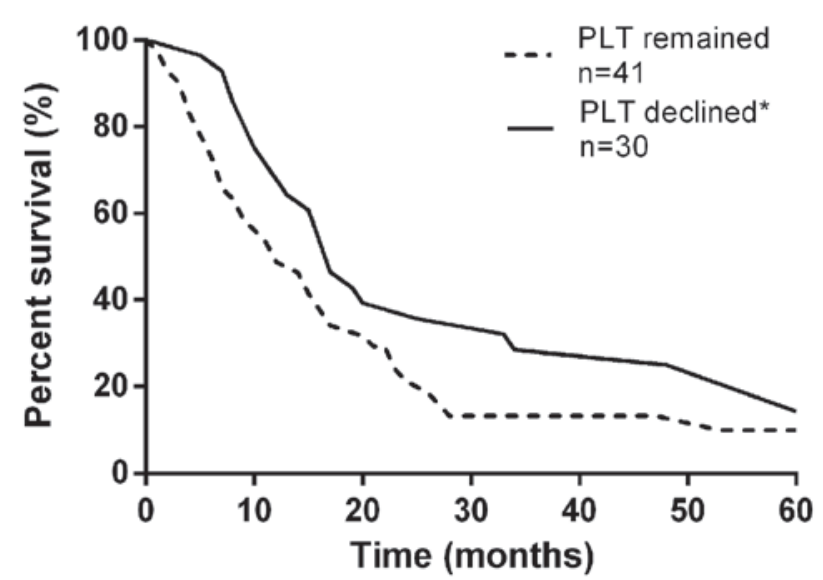

Figure 2. Survival analysis between GC patients with PLT counts that were (A) normal, $>300 \times 10^{9} / 1(\mathrm{P}=0.227)$ and $>400 \times 10^{9} / 1(\mathrm{P}=0.008)$ and $(\mathrm{B})$ decreased to a normal level and remained $>400 \times 10^{9} / 1(\mathrm{P}=0.417)$. PLT, platelet; $\mathrm{GC}$, gastric cancer. 
Table IV. Multivariate analysis of the prognostic indicators.

\begin{tabular}{|c|c|c|c|}
\hline Factors & RR & $95 \% \mathrm{CI}$ & P-value \\
\hline \multicolumn{4}{|l|}{ PLT, $\times 10^{9} / 1$} \\
\hline$\leq 300$ & 1.000 & & \\
\hline$>400$ & 1.538 & $1.041-2.271$ & 0.031 \\
\hline \multicolumn{4}{|c|}{ TNM classification } \\
\hline I & 1.000 & & \\
\hline II & 1.692 & $0.544-5.267$ & 0.364 \\
\hline III & 3.339 & $0.941-11.852$ & 0.062 \\
\hline IV & 6.875 & $1.824-25.914$ & 0.004 \\
\hline \multicolumn{4}{|c|}{ Depth of penetration } \\
\hline $\mathrm{T} 1$ & 1.000 & & \\
\hline $\mathrm{T} 2$ & 0.664 & $0.225-1.954$ & 0.457 \\
\hline $\mathrm{T} 3$ & 0.856 & $0.447-1.641$ & 0.640 \\
\hline $\mathrm{T} 4$ & 0.927 & $0.473-1.816$ & 0.825 \\
\hline \multicolumn{4}{|c|}{ Lymphatic invasion } \\
\hline Absent & 1.000 & & \\
\hline Present & 3.795 & $1.565-9.203$ & 0.003 \\
\hline \multicolumn{4}{|c|}{ Tumor size, $\mathrm{cm}$} \\
\hline$<5$ & 1.000 & & \\
\hline$\geq 5$ & 0.826 & $0.555-1.228$ & 0.344 \\
\hline \multicolumn{4}{|c|}{ Degree of differentiation } \\
\hline Well & 1.000 & & \\
\hline Moderate & 1.685 & $0.808-3.517$ & 0.164 \\
\hline Poor & 1.239 & $0.656-2.344$ & 0.509 \\
\hline
\end{tabular}

P-values were calculated using the Enter method. RR, relative risk; CI, confidence interval; TNM, tumor, nodes, metastasis; PLT, platelet.

with a PLT count $>300 \times 10^{9} / 1$ exhibited no significant difference in prognosis $(\mathrm{P}=0.227)$. The results demonstrated that $\sim 42.25 \%$ (30/71) of patients did not have thrombocytosis following resection, however, the reason was unknown. Due to preoperative PLT counts being maintained at a high level in the most advanced-stage patients who were unable to undergo D2 dissection, the postoperative PLT count was also useful for predicting prognosis ( $\mathrm{P}=0.046$; Fig. 2B).

Prior to multivariate analysis, association analysis between thrombocytosis and clinicopathological features allowed the exclusion of age, location, type, vascular invasion and perineural invasion. Thus, PLT counts, TNM classification, depth of penetration, tumor size, degree of differentiation and lymphatic invasion were evaluated using the Cox proportional hazard model. Of the six factors, PLT count, TNM classification and lymphatic invasion were identified as independent prognostic indicators of survival (Table IV). Individuals with thrombocytosis had a relative risk (RR) for mortality of 1.538 (95\% CI, 1.041-2.271; P=0.031), 1.994 for TNM classification (95\% CI, 1.432-2.777; $\mathrm{P}<0.001)$ and 3.975 for lymphatic invasion (95\%CI, 1.565-9.203; $\mathrm{P}=0.003)$.

Thrombocytosis monitoring for the recurrence of $G C$. To evaluate the role of thrombocytosis $\left(\mathrm{PLT}>400 \times 10^{9} / 1\right)$ in cancer recurrence, differences were compared between tumor patients with a normal PLT count and a group of individuals whose PLT count decreased from $>400 \times 10^{9} / 1$ to normal following surgery. Sensitivities for predicting the recurrence of malignancy in patients with normal and decreased PLT counts were 24.1 and $70.8 \%$, respectively. In addition, the specificities of the two groups were 88.2 and $83.3 \%$, respectively. Therefore, thrombocytosis in cancer patients with a decreased postoperative PLT count had a significant advantage for predicting tumor recurrence with AUROC, as compared with patients that had a normal PLT count prior to surgery ( 0.847 vs. 0.550 ; $\mathrm{P}=0.004$; Table V).

\section{Discussion}

As a multifunctional factor, an abnormal surplus of PLTs is associated with tumor size, TNM classification, invasive degree, prognosis and tumor recurrence in GC, as well as D-dimer and fibrinogen blood concentrations. The association between DVT and thrombocytosis remains to be clarified as ultrasonic testing was only performed on suspected patients that exhibited major complaints. In addition, the decrease in the number of PLTs temporarily following surgery may have been due to the surgical patients suffering blood loss during the procedure. Furthermore, clinical infusion during the fasting period contributed to hemodilution. 
Table V. Comparison of tumor recurrence among PLT levels.

Sensitivities and specificities for recurrence

\begin{tabular}{|c|c|c|c|c|c|c|c|}
\hline Group & PLT, $\times 10^{9} / 1$ & Imaging & $\mathrm{n}$ & Sensitivity, $\%$ & Specificity, $\%$ & AUROC & P-value \\
\hline \multirow[t]{3}{*}{$\begin{array}{l}\text { Normal PLT } \\
(\mathrm{n}=100)\end{array}$} & $>400$ & + & $\begin{array}{r}20 \\
2\end{array}$ & & & & \\
\hline & $\leq 400$ & + & 63 & & & 0.5500 & \\
\hline & & - & 15 & 24.1 & 88.2 & $95 \%$ CI, 408-0.691 & 0.521 \\
\hline \multirow{3}{*}{$\begin{array}{l}\text { Declined PLT } \\
(\mathrm{n}=30)\end{array}$} & $>400$ & + & 17 & & & & \\
\hline & $\leq 400$ & + & $\frac{1}{7}$ & & & $0.847^{\mathrm{a}}$ & \\
\hline & & - & 5 & 70.8 & 83.3 & $95 \%$ CI, $0.707-0.988$ & 0.010 \\
\hline
\end{tabular}

${ }^{a}$ Declined PLT group had a significantly better AUROC value as compared with the normal PLT group ( $\left.\mathrm{P}=0.004\right)$. AUROC, area under the receiver operating characteristic curve; PLT, platelet; CI, confidence interval. Imaging refers to computed tomography scans, + , recurrence is verified.

Malignant tumors with secondary thrombocythemia have been increasingly studied. Without a uniform standard, thrombocytosis has been defined as a PLT count $>400 \times 10^{9} / 1$ in the majority of studies (5-7). However, 220, 300, 350 and $500 \times 10^{9} / 1$ have also been used as the threshold in previous studies $(5,14-16)$. The confusion in the definition of thrombocytosis has caused deviation in experimental results and the reduction of lateral comparability between studies. As demonstrated in the present study, no statistically significant differences were identified between the two cancer groups with PLT counts of $>300 \times 10^{9} / 1$ and $>400 \times 10^{9} / 1$ with regard to the linear correlation between the PLT and leukocyte counts or the associated clinicopathological features. The difference in the linear correlation between hemoglobin concentrations and PLT levels was omitted due to the slight correlation. Survival analysis also illustrated the advantage of using PLT $>400 \times 10^{9} / 1$ as the standard for predicting prognosis. Clinically, there are a number of situations that can cause secondary thrombocytosis in patients with malignant solid tumors, including infection, anemia, inflammation and necrosis. Therefore, it is a reasonable hypothesis to define thrombocytosis in GC patients as those with a PLT count $>400 \times 10^{9} / 1$. This excludes clinical confounding without causing a statistical deviation.

Although speculation remains, several hypotheses have been proposed with regard to the mechanisms by which thrombocytosis develops in malignancies. Released by the liver, kidney and skeletal muscle, thrombopoietin (TPO) specifically stimulates the proliferation and maturation of megakaryocytes, as well as the release of PLTs. In specific cases of tumor-associated thrombocytosis, the concentration of plasmatic TPO significantly increased due to complex pathophysiological factors (17). In addition, activation of the TPO receptor and the feedback regulation of TPO mRNA in the bone marrow participate in this phenomenon (18). However, TPO is not the only factor, as the body is able to produce a few PLTs following TPO gene knockout and the elimination of the TPO receptor (19). Interleukin-1 and -6 are bone marrow-stimulating cytokines that are associated with thrombocytosis and may potentially facilitate the production of PLTs $(20,21)$. As previous studies have shown, the morbidity of thrombocytosis in various malignancies is inhomogeneous. It was found that $\sim 42.5 \%$ of ovarian epithelial cancer cases presented with concurrent thrombocytosis, as well as $56.8 \%$ of renal cell carcinoma cases $(2,22)$. These results are much higher compared with other tumors, where the occurrence of thrombocytosis was $4.0 \%$ in GC patients and $4.5 \%$ in non-small-cell lung cancer patients (23). With regard to histoembryology classification, hematopoietic cells and the urogenital system originate from the same mesoderm, while the digestive and respiratory system originate from the same endoderm. Thus, we hypothesized that compared with the digestive system, the biological function of malignant tumors derived from the urogenital system may be more inclined to thrombocytosis.

Thrombocytosis may adversely affect survival in malignancies by promoting neoplasm invasion, adhesion and proliferation. Tumor cell-induced PLT aggregation may activate and aggregate PLTs to mediate adhesion to cancer cells via glycoproteins-Ib-IX, IIb/IIIa or adenosine diphosphate (24). PLTs efficiently shield and protect malignant cells from the host's immune system and provide a useful medium for the adhesion of cancer cells to the vascular endothelium through forming tumor thrombi and adhesion molecules, including P-selectin and von Willebrand factor $(25,26)$. Following adhesion, PLTs also play a role in tumor growth by secreting several tumor growth and angiogenic factors, including PLT-derived growth factor, arachidonic acid and vascular endothelial growth factor (VEGF). VEGF highly correlates with PLTs as an angiogenic factor and was shown to adversely affect survival in GC $(27,28)$. Neovascularization is necessary for the development of neoplasm, which also explains the association between thrombocytosis and TNM classification, depth of penetration, tumor size and prognosis. However, predicting cancer recurrence with PLTs has been rarely reported. Although the mechanism is unclear, the clinical data indicate the significance of monitoring PLTs in a specific population; patients who suffered thrombocytosis preoperatively, but recovered following surgery.

In conclusion, a PLT count of $400 \times 10^{9} / 1$ is an ideal threshold value for the definition of thrombocytosis. Although 
the incidence $(4.0 \%)$ was lower than other types of cancer, thrombocytosis was shown to be associated with a number of clinicopathological features and function as an independent prognostic indicator and cancer recurrence monitor.

\section{Acknowledgements}

The study was supported by grants from the Zhejiang Provincial Natural Science Foundation of China (no. Y2100660) and the Wenzhou Science and Technology Bureau (no. H20100028).

\section{References}

1. Delhommeau F, Jeziorowska D, Marzac C and Casadevall N: Molecular aspects of myeloproliferative neoplasms. Int J Hematol 91: 165-173, 2010.

2. Gungor T, Kanat-Pektas M, Sucak A and Mollamahmutoglu L: The role of thrombocytosis in prognostic evaluation of epithelial ovarian tumors. Arch Gynecol Obstet 279: 53-56, 2009.

3. Huang Z, Jiang C, Zhang YM and Yao B: Analysis of hemogram, iron staining in bone marrow and iron metabolism in 98 patients with thrombocytosis. Chinese Journal of Clinical Laboratory Science 26: 49-51, 2008.

4. Tchebiner JZ, Nutman A, Boursi B, et al: Diagnostic and prognostic value of thrombocytosis in admitted medical patients. Am J Med Sci 342: 395-401, 2011.

5. Iwasaki A, Hamanaka W, Harnada T, Maekawa S, Enatsu S and Shirakusa T: Significance of platelet counts in patients who underwent surgical treatment for lung metastasis. Int Surg 92: 103-109, 2007.

6. Ikeda M, Furukawa H, Imamura H, et al: Poor prognosis associated with thrombocytosis in patients with gastric cancer. Ann Surg Oncol 9: 287-291, 2002.

7. Maráz A, Furák J, Varga Z, Kahán Z, Tiszlavicz L and Hideghéty K: Thrombocytosis has a negative prognostic value in lung cancer. Anticancer Res 33: 1725-1729, 2013.

8. Gouin-Thibault I, Achkar A and Samama MM: The thrombophilic state in cancer patients. Acta Haematol 106: 33-42, 2001.

9. Riess L: Pathology of the blood. Arch Anat Physiol Wissensch Med 39: 237-249, 1872 (In German).

10. Ferrigno D and Buccheri G: Hematologic counts and clinical correlates in 1201 newly diagnosed lung cancer patients. Monaldi Arch Chest Dis 59: 193-198, 2003.

11. Qiu MZ, Xu RH, Ruan DY, et al: Incidence of anemia, leukocytosis, and thrombocytosis in patients with solid tumors in China. Tumour Biol 31: 633-641, 2010.

12. Aminian A, Karimian F, Mirsharifi R, et al: Significance of platelet count in esophageal carcinomas. Saudi J Gastroenterol 17: 134-137, 2011.

13. Aoe K, Hiraki A, Ueoka H, et al: Thrombocytosis as a useful prognostic indicator in patients with lung cancer. Respiration 71: $170-173,2004$
14. Brockmann MA, Giese A, Mueller K, et al: Preoperative thrombocytosis predicts poor survival in patients with glioblastoma. Neuro Oncol 9: 335-342, 2007.

15. Nather A, Mayerhofer K, Grimm C, et al: Thrombocytosis and anaemia in women with recurrent ovarian cancer prior to a second-line chemotherapy. Anticancer Res 23: 2991-2994, 2003.

16. Zwitter M, Kovac V, Smrdel U, Kocijancic I, Segedin B and Vrankar M: Phase I-II trial of low-dose gemcitabine in prolonged infusion and cisplatin for advanced non-small cell lung cancer. Anticancer Drugs 16: 1129-1134, 2005.

17. Cerutti A, Custodi P, Duranti M, Noris P and Balduini CL: Thrombopoietin levels in patients with primary and reactive thrombocytosis. Br J Haematol 99: 281-284, 1997.

18. Soonthornthum T, Suraseraneewong V, Kengsakol K, Wijaithum K, Kasemsan P and Prommatt S: Thrombocytosis in advanced epithelial ovarian cancer. J Med Assoc Thai 90: 1495-1500, 2007

19. de Sauvage FJ, Carver-Moore K, Luoh SM, et al: Physiological regulation of early and late stages of megakaryocytopoiesis by thrombopoietin. J Exp Med 183: 651-656, 1996.

20. Lidor YJ, Xu FJ, Martínez-Maza O, et al: Constitutive production of macrophage colony-stimulating factor and interleukin-6 by human ovarian surface epithelial cells. Exp Cell Res 207: 332-339, 1993.

21. Ceresa IF, Noris P, Ambaglio C, Pecci A and Balduini CL: Thrombopoietin is not uniquely responsible for thrombocytosis in inflammatory disorders. Platelets 18: 579-582, 2007.

22. Symbas NP, Townsend MF, El-Galley R, Keane TE, Graham SD and Petros JA: Poor prognosis associated with thrombocytosis in patients with renal cell carcinoma. BJU Int 86: 203-207, 2000.

23. Tomita M, Shimizu T, Ayabe T and Onitsuka T: Prognostic significance of the combined use of preoperative platelet count and serum carcinoembryonic antigen level in non-small-cell lung cancer. Gen Thorac Cardiovasc Surg 58: 573-576, 2010.

24. Lian L, Li W, Li ZY, et al: Inhibition of MCF-7 breast cancer cell-induced platelet aggregation using a combination of antiplatelet drugs. Oncol Lett 5: 675-680, 2013.

25. Palumbo JS, Talmage KE, Massari JV, et al: Platelets and fibrin(ogen) increase metastatic potential by impeding natural killer cell-mediated elimination of tumor cells. Blood 105: $178-185,2005$

26. McCarty OJ, Mousa SA, Bray PF and Konstantopoulos K: Immobilized platelets support human colon carcinoma cell tethering, rolling, and firm adhesion under dynamic flow conditions. Blood 96: 1789-1797, 2000.

27. Bachelot T, Ray-Coquard I, Menetrier-Caux C, Rastkha M, Duc A and Blay JY: Prognostic value of serum levels of interleukin 6 and of serum and plasma levels of vascular endothelial growth factor in hormone-refractory metastatic breast cancer patients. Br J Cancer 88: 1721-1726, 2003.

28. Wang X, Chen X, Fang J and Yang C: Overexpression of both VEGF-A and VEGF-C in gastric cancer correlates with prognosis, and silencing of both is effective to inhibit cancer growth. Int J Clin Exp Pathol 6: 586-597, 2013. 